\title{
A Novel Approach to Treatment of Pinguecula Using Atmospheric Low-temperature Plasma: A Clinical Case Series
}

\author{
Farhad Nejat" ${ }^{*}$ Khosrow Jadidi, Mohammad Amin Nejat, Nazanin-Sadat Nabavi, \\ Seyede-Yasamin Adnani, Shima Eghtedari
}

Vision Health Research Center, Semnan University of Medical Sciences, Semnan, Iran

Email address:

fanejat@yahoo.com (F. Nejat)

${ }^{*}$ Corresponding author

\section{To cite this article:}

Farhad Nejat, Khosrow Jadidi, Mohammad Amin Nejat, Nazanin-Sadat Nabavi, Seyede-Yasamin Adnani, Shima Eghtedari. A Novel Approach to Treatment of Pinguecula Using Atmospheric Low-temperature Plasma: A Clinical Case Series. American Journal of Clinical and Experimental Medicine. Vol. 9, No. 5, 2021, pp. 142-146. doi: 10.11648/j.ajcem.20210905.13

Received: August 26, 2021; Accepted: September 13, 2021; Published: September 30, 2021

\begin{abstract}
Background: In recent years plasma medicine, played an important role in treating several ocular surface disorders. This study will introduce atmospheric low-temperature plasma (ALTP) for removing the pinguecula. Methods: In the clinical case series, five eyes of five patients ( 2 patients OD, 3 patients OS) with pinguecula were included. The pinguecula removed and completely healed, using the white handpiece of the plasma generator device (Plexr, GMV s.r.l Grottaferrata, Italy). Refraction parameters, uncorrected distance visual acuity (UDVA) and corrected distance visual acuity (BDVA), intraocular pressure (IOP), corneal fluorescein staining (CFS), contrast sensitivity (CS) and dry eye tests including, tear meniscus height (TMH) and tear breakup time (TBUT) were measured before, one month and six months after treatment for all patients. Results: The median size of the pinguecula was $3.5 \mathrm{~mm}$ (ranged from 2.5 to $4.5 \mathrm{~mm}$ ). In all patients, the pinguecula was completely sublimated and after six months of follow-up, no evidence of recurrence was observed. In addition, the patient follow-up did not reveal any considerable complications during or 6 months after the surgery. Conclusion: Overall, the current study showed that, plasma assisted noninvasive surgery (PANIS method) is a simple, office-based, minimally invasive, effective approach to treat pinguecula with no considerable complications but it is noteworthy that, study with higher sample size like clinical trial is needed.
\end{abstract}

Keywords: Pinguecula, Sublimation, Soft Surgery, PANIS Method, Atmospheric Low-temperature Plasma

\section{Introduction}

A Pinguecula is a common conjunctival condition that occurs typically on the nasal side of the bulbar conjunctiva, adjacent to the limbus in the interpalpebral zone. It is usually bilateral, appears as a yellow-white elevated mass, and occurs because of the effects of aging, UV light exposure and environmental insults such as dust and wind. Pinguecula represent an elastotic degeneration of subepithelial collagen with hyalinized connective tissue. The mass may enlarge gradually over long periods of time [1]. Pinguecula usually does not cause any serious problems for patient and needs no treatment nevertheless, in some cases, it may be associated with an inflammatory reaction and present with symptoms such as foreign body sensation, itching, burning, tearing, and redness $[2,3]$. Moreover, due to the inability for using contact lenses or cosmetic concerns, the individual may want to have it removed $[4,5]$. Although limited studies have reported the prevalence of pinguecula, the available evidence suggests that the prevalence of pinguecula in the adult population can be as high as $69.5 \%$ [6]. In addition, the results of two population-based studies in Shahroud and Tehran have estimated the prevalence of this lesion at approximately $60 \%$ in the population aged over 40 years old in Iran [7, 8].

Usually, surgery is considered as one of the elective approaches toward treating and removing this lesion, however, issues such as wound, scarring, conjunctival bleeding, and recurrence can complicate conventional surgeries $[9,10]$. Furthermore, the use of conventional surgical methods may be requires the presence of the person in the operation room, 
anesthesia, and suturing. One approach that has been employed for less invasive surgeries is the utilization of the plasma based soft surgery in the medical field [11]. The applications of plasma in medicine are in destroying tumor cells, disinfection, dentistry, dermatology, wound healing, pharmacology, and the other applications that are added to the list nowadays [11-14]. In this regard, several plasma sources approved for medical purposes. In addition to its less-invasive nature, reduced healing duration, reduced treatment costs, and reduced complications are among other advantages of this technique. Given the potential of plasma in soft tissue surgeries, Nejat et al. have evaluated the safety of this method in ophthalmic field for the first time in 2018. The results of this study that has been conducted at experimental level indicated that the use of atmospheric low temperature plasma (ALTP) on the rabbit's conjunctiva is harmless, and this type of plasma can be used as an alternative procedure to treatment of some of the ocular surface disorders [15].

Thus, given that to our knowledge, no study has published the use of plasma in treating pinguecula so we conducted this study to introduce the plasma based soft surgery procedure for removing the pinguecula.

\section{Methods}

\subsection{Participants}

The current study is a clinical case series conducted in 2019 in the clinic of the Vision Health Research Center of Semnan University of Medical Sciences. The study population consisted of patients with pinguecula. All the patients who had symptoms such as irritation, tearing, foreign body sensation, dryness and burning were diagnosed with pinguecula possessed the inclusion criteria. In addition, exclusion criteria was history of ocular surgery in the past six months. Finally, five patients with pinguecula were included in this study. After completely describing the objectives of the study, the plasma treatment procedure and its probable risks, and other treatment options for this disorder, written informed consent was taken from all the participants. The Ethics Committee of Semnan University of Medical Sciences under ethics code IR. REC. 1398.318 approved this study.

\subsection{Measurements}

Before treatment, all the patients were examined for the following: refractive errors, uncorrected distance visual acuity (UDVA), corrected distance visual acuity (CDVA), intraocular pressure (IOP), contrast sensitivity (CS). IOP, CS and aberrometry were performed using the rebound tonometer (Icare Finland Oy, Vantaa, Finland) and Mars letter contrast sensitivity test (Mars Perceptrix, Chappaqua, New York, USA), respectively.

Corneal fluorescein staining (CFS) and dry eye tests, including, tear meniscus height (TMH) and tear breakup time (TBUT). TBUT, TMH, and CFS were measured using a handheld ocular surface analyzer (OSA-VET, SBM Sistemi, Torino, Italy).

All the aforementioned parameters measured one month and six months after the treatment too. Dry eye and its impact on vision assessed with the ocular surface disease index (OSDI) questionnaire. This questionnaire is a rapid assessment of ocular stimuli related to eye dryness and its impact on vision-related performance [16]. A higher OSDI score indicates dryness and greater patient dissatisfaction.

All the patients were followed one month and six months after the surgery for symptoms of burning, itching, foreign body sensation, dry eye, tearing and satisfaction.

\subsection{Intervention (Plasma Assisted Non-invasive Surgery (PANIS))}

The plasma generator device (Plexr, GMV s.r.l Grottaferrata, Italy) was used to remove the pinguecula. The plexr generates plasma by ionizing the air between the tip of the device and the target tissue. However; in this study, we used a white handpiece plexr that produces the plasma with the least amount of power ( 0.7 watts) (table 1$)$.

Table 1. Technical features of the Plexr device.

\begin{tabular}{ll}
\hline Parameters & Values \\
\hline Working gas Air & Air \\
Power supply & Docking station $=24 \mathrm{~V}$ \\
& Hand pieces: embedded inductive charger $=5 \mathrm{~V}$ \\
Hand pieces: & $\leq 2 \mathrm{~W}$ \\
Max output & $\leq 1.3 \mathrm{kVPP}$ \\
Max working voltage & $(70-80) \mathrm{kHz}$ \\
$\begin{array}{l}\text { Output frequency } \\
\text { Hand piece types: }\end{array}$ & V peak to peak $=500 \mathrm{~V}$, Power $=0.7 \mathrm{~W}$, Frequency $=75 \mathrm{kHz}$ \\
White* & V peak to peak $=600$ V, Power $=1 \mathrm{~W}$, Frequency $=75 \mathrm{kHz}$ \\
Green & V peak to peak $=700$ V, Power $=2 \mathrm{~W}$, Frequency $=75 \mathrm{kHz}$ \\
Red & $120 \mathrm{~W}$ \\
Maximum absorbed power & \\
(Docking station) & Stainless steel sterile disposable needle \\
Applicator electrode & Ilb**(Medium-high risk) \\
Risk classification of the device & \\
\hline
\end{tabular}

* In current study, the white hand piece was used.

**This classification relates to the Non-invasive medical devices within the field of dermatology. 
First, the patient's eye was anesthetized with topical Tetracaine $0.5 \%$ eye drops (Sina Darou, Tehran, Iran). The plasma-assisted non-invasive surgery (PANIS) method done when patients being placed behind the slit lamp. The surgeon apply a spot to the top of the pinguecula bump with plexr device. Then other spots are applied continuously until the bump was completely sublimated and to the point that the sclera was undamaged. One surgeon (Dr. F. Nejat) did all the surgeries.

As the post-op medications, patients were given a prescription containing chlobiotic $0.5 \%$ (Sina Darou, Tehran, Iran) every six hours and betamethasone $0.1 \%$ eye drops (Sina Darou, Tehran, Iran) every four hours for a week. The dose of betamethasone tapered off over one month (one drop every 6 , 8 , and 12 hours during the second, third, and fourth week, respectively). All methods were performed in accordance with the relevant guidelines and regulations.

\section{Results}

Overall, this treatment procedure was performed on five patients with pinguecula; three of them were men and the other two were women. The youngest patient was 30 and the eldest was 53 years old. The median size of the pinguecula was $3.5 \mathrm{~mm}$ (ranged from 2.5 to $4.5 \mathrm{~mm}$ ). The patient follow-up did not reveal any considerable complications during or after the surgery. In all patients, the pinguecula was completely sublimated and after six months of follow-up, no evidence of recurrence was observed. Table 2 shows the patients' characteristics, lesion size and position, visual parameters, and dry eye tests. Based on these results, the patients' OSDI scores had a declining trend in the patients in different follow-ups, such that the mean OSDI score decline from 18.51 before treatment to 5.05 in six months after treatment. As shown in table 2, corneal fluorescein staining improved in three patients.

Table 2. Patients Characteristics and Parameters Measured in Different Examinations.

\begin{tabular}{|c|c|c|c|c|c|c|c|c|c|c|c|c|}
\hline \multirow{2}{*}{$\begin{array}{l}\text { Examination } \\
\text { time }\end{array}$} & \multirow{2}{*}{$\begin{array}{l}\text { UDVA } \\
\text { (Decimal) }\end{array}$} & \multicolumn{3}{|c|}{ Refraction } & \multirow{2}{*}{$\begin{array}{l}\text { CDVA } \\
\text { (Decimal) }\end{array}$} & \multirow{2}{*}{$\begin{array}{l}\text { IOP } \\
(\mathrm{mmHg})\end{array}$} & \multirow{2}{*}{$\begin{array}{l}\text { TBUT } \\
\text { (s) }\end{array}$} & \multirow{2}{*}{$\begin{array}{l}\text { TMH } \\
(\mathbf{m m})\end{array}$} & \multirow{2}{*}{$\begin{array}{l}\text { CFS } \\
\text { grade }^{\dagger}\end{array}$} & \multirow{2}{*}{$\begin{array}{l}\text { Schirmer } \\
(\mathrm{mm})\end{array}$} & \multirow{2}{*}{$\begin{array}{l}\text { OSDI } \\
\text { score* }\end{array}$} & \multirow{2}{*}{$\begin{array}{l}\text { CS } \\
(\log M A R)\end{array}$} \\
\hline & & Sphere & Cylinder & Axis & & & & & & & & \\
\hline \multicolumn{13}{|c|}{ Case 1: Female (30-year-old), Ping site: Nasal, Ping size: 1 x $2.5 \mathrm{~mm}$} \\
\hline Preop. & 1 & -0.25 & 0 & 0 & 1 & 13 & 7 & 0.1 & 0 & 10 & 27.27 & 1.76 \\
\hline 1 month postop. & 1 & -0.25 & -0.25 & 150 & 1 & 14 & 7 & 0.2 & 0 & 12 & 5 & 1.76 \\
\hline 6 months postop. & 1 & 0 & -0.25 & 130 & 1 & 10 & 9 & 0.1 & 0 & 8 & 0 & 1.76 \\
\hline \multicolumn{13}{|c|}{ Case 2: Male (47-year-old), Ping site: Nasal, Ping size: 3 x $3 \mathrm{~mm}$} \\
\hline Preop. & 1 & 0 & -0.5 & 90 & 1 & 12 & 10 & 0.2 & 0 & 10 & 16.66 & 1.52 \\
\hline 1 month postop. & 1 & 0 & -0.5 & 100 & 1 & 10 & 11 & 0.1 & 0 & 10 & 12.5 & 1.52 \\
\hline 6 months postop. & 1 & 0 & -0.5 & 90 & 1 & 11 & 14 & 0.2 & 0 & 12 & 12.5 & 1.52 \\
\hline \multicolumn{13}{|c|}{ Case 3: Male (49-year-old), Ping site: Nasal, Ping size: 3 x $4.5 \mathrm{~mm}$} \\
\hline Preop. & 1 & 0 & 0 & 0 & 1 & 8 & 10 & 0.1 & I & 5 & 18.18 & 1.68 \\
\hline 1 month postop. & 1 & 0 & 0 & 0 & 1 & 9 & 5 & 0.1 & 0 & 5 & 0 & 1.68 \\
\hline 6 months postop. & 1 & 0 & 0 & 0 & 1 & 9 & 10 & 0.1 & 0 & 3 & 0 & 1.68 \\
\hline \multicolumn{13}{|c|}{ Case 4: Female (46-year-old), Ping site: Nasal, Ping size: $1.5 \times 4 \mathrm{~mm}$} \\
\hline 1 month postop. & 1 & 0.5 & 0 & 0 & 1 & 12 & 12 & 0.2 & 0 & 10 & 10 & 1.68 \\
\hline 6 months postop. & 1 & 0.5 & -0.5 & 55 & 1 & 14 & 9 & 0.1 & 0 & 7 & 4.5 & 1.68 \\
\hline \multicolumn{13}{|c|}{ Case 5: Male (53-year-old), Ping site: Nasal, Ping size: 2.5 × 3.5} \\
\hline Preop. & 1 & 0.25 & 0 & 0 & 1 & 15 & 13 & 0.2 & I & 12 & 20.45 & 1.44 \\
\hline 1 month postop. & 1 & 0.25 & 0 & 0 & 1 & 17 & 12 & 0.1 & I & 10 & 2.27 & 1.64 \\
\hline 6 months postop. & 1 & 0 & 0 & 0 & 1 & 15 & 15 & 0.1 & 0 & 10 & 2.27 & 1.64 \\
\hline
\end{tabular}

Accordingly, all patients were satisfied with the removal of the pinguecula. Figures 1 to 5 show the status of the pinguecula lesion before intervention and 6 months post-operation.

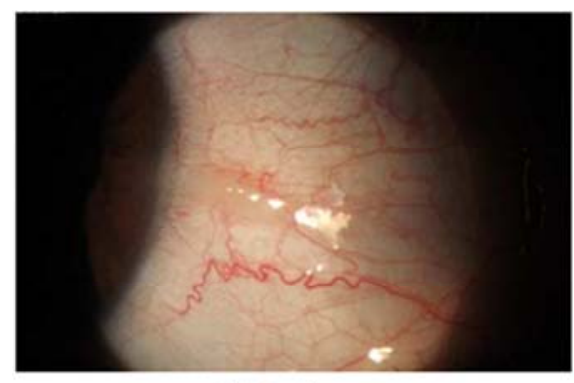

(A) before

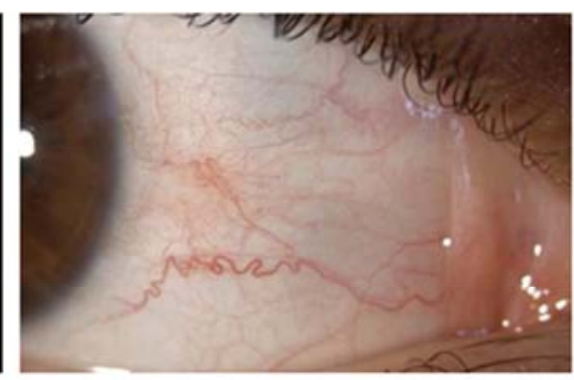

(B) six months after treatment

Figure 1. Slit-lamp images of Patient 1 (A) before (B) six months after treatment. 


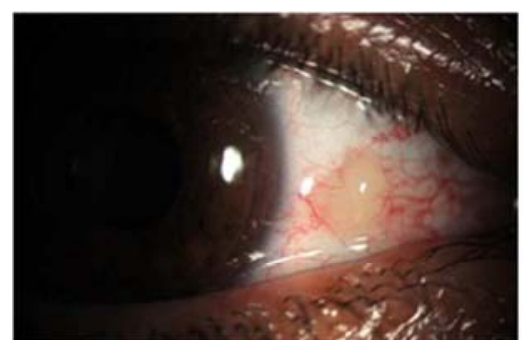

(A) before

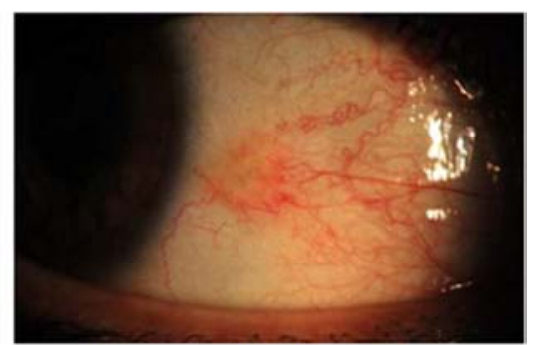

(B) six months after treatment

Figure 2. Slit-lamp images of Patient 2 (A) before (B) six months after treatment.

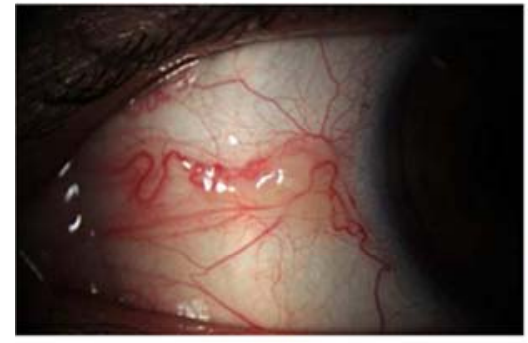

(A) before

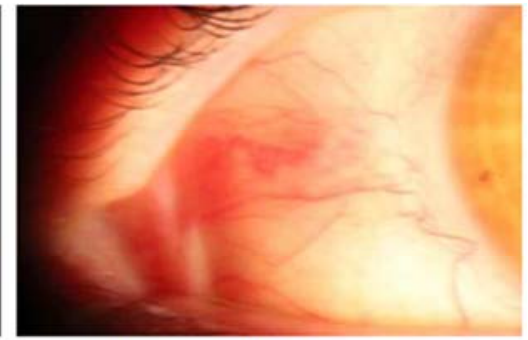

(B) six months after treatment

Figure 3. Slit-lamp images of Patient 3 (A) before (B) six months after treatment.

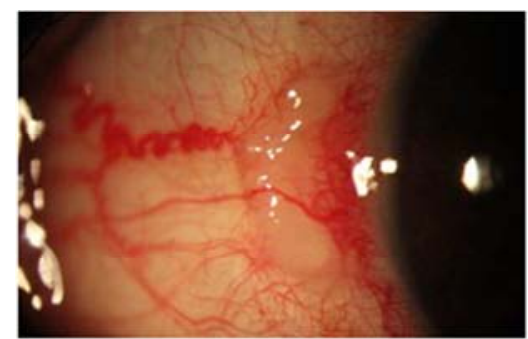

(A) before

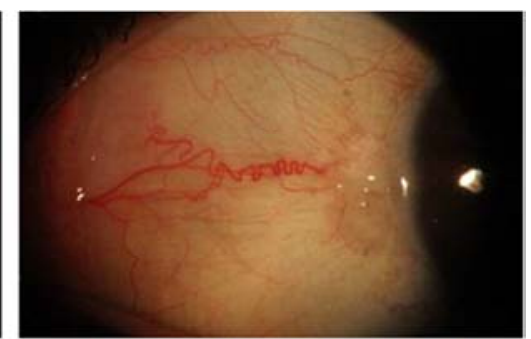

(B) six months after treatment

Figure 4. Slit-lamp images of Patient 4 (A) before (B) six months after treatment.

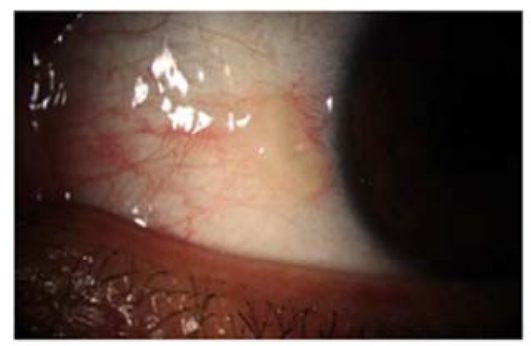

(A) before

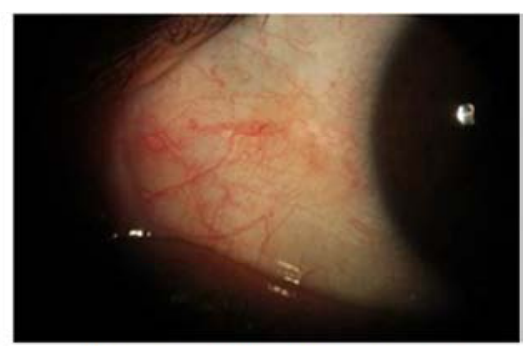

(B) six months after treatment

Figure 5. Slit-lamp images of Patient 5 (A) before (B) six months after treatment.

\section{Discussion}

Pinguecula is a degenerative conjunctival lesion that is usually symptomless, does not cause any serious problems for the affected individual [17]. Based on literature, in many cases the pinguecula does not require surgical excision. Thus, fewer studies have discussed its methods of treatment [18, 19]. Nevertheless, in addition to cosmetic concerns, in certain cases the pinguecula can be associated with attacks of redness, pain, burning, and foreign body sensation, and even its growth towards the limbus that can cause complaints that are more serious for the individual $[2,3]$. To our knowledge, this is the first study at the human level to introduce the plasma based soft surgery procedure for removing the pinguecula. According to our results, in the six-month follow-up period of patients, serious complications was not observed. Moreover, none of the patients reported considerable pain during or after the surgery. Also, despite the complete removal of the lesion, no damage was observed in the deeper tissues.

In a historical study, Seong Joon Ahn et al compared the performance of photocoagulation vs. surgical excision in the treatment of pinguecula. In this study, 21 eyes treated with argon laser photocoagulation, and 23 eyes treated with surgical excision. Bleeding and scarring were reported by both of the approaches. Nevertheless, subconjunctival bleeding and 
scarring in the photocoagulation group were less frequently observed than in the surgical excision group [9].

E. Arenas-Archila et al. in a study with two clinical cases showed the injectionof intralesional betamethasone might be produced significant clinical improvement in symptomatic pinguecula patients. Although during the follow-up period no considerable side effects were reported, it should be noted that the follow-up period is relatively short and it is not possible to observe long-term side effects [18].

Previous evidence showed "pinguecula excision and conjunctival autograft using fibrin glue is an effective and safe approach to the treatment of pinguecula" [10]. These studies reported that surgical excision of symptomatic pinguecula improved not only cosmesis but also improved dry eye syndrome and vascular regression without any recurrence or other considerable complications $[5,10]$.

The PANIS method has certain advantages, the most important of which are its simplicity and low cost. This method is less invasive than the other commonly employed surgical methods and can be performed in the clinic without the need for an operating room and behind a slit lamp. Furthermore, based on our earlier study's results, the use of plasma does not damage the deeper tissues.

In spite of the promising results, our results had certain limitations that should be taken into account while interpreting them. This was a case series that was conducted with a few patients and six-month follow-up. Based on this small number we cannot make concrete conclusions on the effects of the PANIS method. Concrete conclusions require studies with greater sample sizes, with control group and longer follow-up time. Nevertheless, in the current study, in addition to successful treatment of the individuals, their follow-up also indicated that, this approach is completely safe and effective.

\section{Conclusion}

Overall, the current study showed that PANIS method can be used to treat pinguecula as a soft and minimally invasive surgical method with no considerable complications. Although more studies with higher sample sizes and longer follow-up periods need to be conducted, this therapeutic method has the potential to be used as a substitute in treating pinguecula without the usual surgical measures.

\section{Acknowledgements}

We are so grateful from the staff of the Vision Health Research Center, Semnan, Iran, for their contribution.

\section{References}

[1] Weisenthal, R. W., et al., External disease and cornea. 2019: American Academy of Ophthalmology.
[2] Mimura, T., et al., Severity and determinants of pinguecula in a hospital-based population. Eye \& Contact Lens, 2011. 37 (1): p. 31-35.

[3] Frucht-Pery, J., et al., Treatment of inflamed pterygium and pinguecula with topical indomethacin $0.1 \%$ solution. Cornea, 1997. 16 (1): p. 42-47.

[4] Mimura, T., et al., Pinguecula and contact lenses. Eye, 2010. 24 (11): p. 1685-1691.

[5] Jeong, J., et al., The Improvement of Dry Eye Symptoms after Pinguecula Excision and Conjunctival Autograft with Fibrin Glue. Journal of ophthalmology, 2019. 2019.

[6] Panchapakesan, J., F. Hourihan, and P. Mitchell, Prevalence of pterygium and pinguecula: the Blue Mountains Eye Study. Australian and New Zealand journal of ophthalmology, 1998. 26: p. S2-S5.

[7] Fotouhi, A., et al., Prevalence and risk factors of pterygium and pinguecula: the Tehran Eye Study. Eye, 2009. 23 (5): p. 1125-1129.

[8] Rezvan, F., et al., The prevalence and determinants of pterygium and pinguecula in an urban population in Shahroud, Iran. Acta Medica Iranica, 2012: p. 689-696.

[9] Ahn, S. J., et al., One-year outcome of argon laser photocoagulation of pinguecula. Cornea, 2013. 32 (7): p. 971-975.

[10] Jung, S., et al., Vascular regression after pinguecula excision and conjunctival autograft using fibrin glue. Eye \& contact lens, 2017. 43 (3): p. 199-202.

[11] Laroussi, M., Plasma medicine: a brief introduction. Plasma, 2018. 1 (1): p. 47-60.

[12] Claiborne, D., et al., Low-temperature atmospheric pressure plasma enhanced tooth whitening: The next-generation technology. International Journal of Dental Hygiene, 2014. 12 (2): p. 108-114.

[13] Shashurin, A., et al., Living tissue under treatment of cold plasma atmospheric jet. Applied Physics Letters, 2008. 93 (18): p. 181501.

[14] Zimmermann, J. L., et al., Disinfection Through Different Textiles Using Low-temperature Atmospheric Pressure Plasma. Plasma Processes and Polymers, 2012.9 (8): p. 792-798.

[15] Nejat, F., et al., Safety evaluation of the plasma on ocular surface tissue: An animal study and histopathological findings. Clinical Plasma Medicine, 2019. 14: p. 100084.

[16] Schiffman, R. M., et al., Reliability and validity of the ocular surface disease index. Archives of ophthalmology, 2000. 118 (5): p. 615-621.

[17] Bell, A., Pinguecula. Journal of Visual Communication in Medicine, 2006. 29 (2): p. 82-83.

[18] Arenas-Archila, E., K. Arellano, and D. Muñoz-Sarmiento, Intra-lesional injection of betamethasone for the treatment of symptomatic pinguecula. Archivos de la Sociedad Española de Oftalmología (English Edition), 2014. 89 (10): p. 408-410.

[19] Somnath, A. and K. Tripathy, Pinguecula. StatPearls [Internet], 2020. 